\title{
Sortilin associates with Trk receptors to enhance anterograde transport and signaling by neurotrophins
}

\author{
Christian B. Vaegter ${ }^{1}$, Pernille Jansen ${ }^{1}$, Anja W. Fjorback ${ }^{2}$, Simon Glerup ${ }^{1}$, Sune Skeldal ${ }^{1}$, \\ Mette Richner ${ }^{1}$, Bettina Erdmann ${ }^{3}$, Jens R. Nyengaard ${ }^{2}$, Lino Tessarollo ${ }^{4}$, Gary R. Lewin ${ }^{3}$, \\ Thomas E. Willnow ${ }^{3}$, Moses V. Chao ${ }^{5}$, and Anders Nykjaer ${ }^{1,6}$ \\ 1)The Lundbeck Foundation Research Center MIND, Department of Medical Biochemistry, Ole \\ Worms Allé 1170, Aarhus University, DK-8000 Aarhus C, Denmark \\ 2)MIND Center, Stereology and Electron Microscopy Laboratory, Aarhus University, 8000 Aarhus \\ C, Denmark \\ 3)Max-Delbrück-Center for Molecular Medicine, 13125 Berlin, Germany \\ 4)Center for Cancer Research, National Cancer Institute, Frederick, Maryland 21702, USA \\ 5)Kimmel Center at Skirball Institute of Biomolecular Medicine, New York University School of \\ Medicine, New York 10016, USA \\ 6) Neuronlcon, Gustav Wieds vej 10, DK-8000 Aarhus C, Denmark
}

\begin{abstract}
Binding of target-derived neurotrophins to Trk receptors at nerve terminals are required to stimulate neuronal survival, differentiation, innervation and synaptic plasticity. The distance between the soma and nerve terminal is tremendous, making efficient anterograde Trk transport critical for their synaptic translocation and signaling. The mechanism responsible for this trafficking remains poorly understood. Here we show that the sorting receptor sortilin interacts with TrkA, -B, and -C and enables their anterograde axonal transport, thereby enhancing neurotrophin signaling. Cultured DRG neurons lacking sortilin exhibit blunted MAPK signaling and reduced neurite outgrowth upon stimulation with NGF. Moreover, deficiency for sortilin considerably aggravates TrkA, -B- and -C phenotypes present in $\mathrm{p} 75^{\mathrm{NTR}}$ knockouts, and results in increased embryonic lethality and sympathetic neuropathy in mice heterozygous for TrkA. Our findings demonstrate a novel and unexpected role for sortilin as an anterograde trafficking receptor for Trk and a positive modulator of neurotrophin-induced neuronal survival.
\end{abstract}

\begin{abstract}
Neurotrophins (NT) are growth factors that are essential to regulate wiring of the nervous system during development, to ensure its maintenance in the adult organism, and to modulate synaptic transmission ${ }^{1}$. The growth factor family comprises nerve growth factor (NGF), brain-derived neurotrophic factor (BDNF), and neurotrophin 3 (NT-3) and -4 (NT-4), respectively, and they exert their trophic effects via binding to members of the tropomyosin-related kinase (Trk) receptors at the axon terminals of innervating neurons. NGF binds most specifically to TrkA, BDNF and NT-4 to TrkB, and NT-3 predominantly to TrkC but co-expression of the structurally unrelated p $75^{\mathrm{NTR}}$ neurotrophin receptor refines the fidelity of the NTs towards their cognate Trks, thereby augmenting trophic responses ${ }^{1}$.
\end{abstract}

Correspondance: Anders Nykjaer, an @ biokemi.au.dk MIND Center, Department of Medical Biochemistry Ole Worms Allé, Bldg. 1170 Aarhus University DK-8000C Aarhus Denmark Fax: +45 86131160 Phone: +45 8942 2884; Christian B. Vaegter, cv@ biokemi.au.dk MIND Center, Department of Medical Biochemistry Ole Worms Allé, Bldg. 1170 Aarhus University DK-8000C Aarhus Denmark. 
Trk receptors are produced at the cell body and rapidly propelled along the axonal shaft to the synapse by a process referred to as anterograde transport. Following NT-mediated activation, the receptors shuttle back from the axon terminals to the soma by retrograde transport in so-called signaling endosomes to regulate gene expression and exert their trophic responses ${ }^{2}$. While our understanding of the retrograde trafficking process is becoming increasingly clear, surprisingly little is known about the mechanisms that underlie anterograde Trk transport. It is well established that axonal transport relies upon microtubule-based motor kinesin-proteins ${ }^{3}$, an interaction that, at least for TrkB, can be mediated by an adaptor complex comprising Slp1/Rab27B and CRMP- $2^{4}$. Yet, knockdown of any of these adaptors only reduces axonal targeting by 30-50\% suggesting the existence of additional transport mechanisms 5 .

Sortilin is one of five members of the Vps10p-domain family of sorting receptors ${ }^{6}$. It is abundantly expressed in neurons of the central as well peripheral nervous systems. While only $\sim 10 \%$ of sortilin is surface exposed, $\sim 90 \%$ of the receptors are located in the TGN, endosomes, dendrites, axons, immature secretory granules, and synaptic vesicles ${ }^{6-10}$. It is now well established that sortilin is capable of both rapid internalization, Golgi to endosome trafficking, retrograde transport to the TGN, and sorting into the pathway for regulated secretion; transport activities that are governed by binding of specific cytosolic adaptor proteins to the intracellular tail of the receptor ${ }^{7,8,10}$. Sortilin is synthesized as an inactive precursor, pro-sortilin, that is incapable of ligand binding due to a propeptide that prevents ligands from entering the binding pocket ${ }^{11}$. In the trans-Golgi network (TGN) proconvertase liberates this propeptide, rendering the receptor active ${ }^{12}$.

We previously described that sortilin can form a tripartite complex with $\mathrm{p} 75^{\mathrm{NTR}}$ and secreted precursor forms of NTs, denoted pro-neurotrophins (proNT), to induce cell death $^{13,14}$. Because only $5-10 \%$ of sortilin is surface exposed and prior studies have demonstrated that this receptor is engaged in cellular sorting activities, we hypothesized that sortilin might also promote anterograde transport of Trks to the nerve endings. In accordance with this hypothesis we here report that sortilin surprisingly also facilitates trophic signaling by ensuring adequate Trk receptor expression at the synapse for mature NTs to stimulate neuronal survival and differentiation.

\section{Results}

\section{Sortilin physically interacts with Trk receptors}

Immunofluorescence staining revealed that sortilin is frequently co-expressed with Trk and p $75^{\mathrm{NTR}}$ in tissues, such as the adult dorsal root ganglia (DRG), which are not destined for apoptosis (Fig. 1a). This observation suggests additional functions for sortilin in neurotrophin action besides induction of apoptosis. Because sortilin is capable of intracellular sorting, we hypothesized that it might affect trafficking and the subcellular distribution of Trk receptors. To test this hypothesis, 293 human embryonic kidney (HEK) cells transfected with cDNA constructs encoding sortilin and TrkA, -B and -C, respectively, were subjected to co-immunoprecipitation experiments using a pan-anti-Trk antibody. We found that sortilin can physically interact with all Trks (Fig. 1b, TrkB data in HEK cells not shown). Importantly, in addition to the mature $140 \mathrm{kDa}$ TrkA receptor, also the immature and incompletely glycosylated $110 \mathrm{kDa}$ precursor bound sortilin ${ }^{15}$ (Fig. 1b).

A robust receptor-receptor interaction was validated in intact cells by fluorescence resonance energy transfer (FRET) and fluorescence lifetime imaging microscopy (FLIM). We obtained a mean apparent pFRET efficiency Eapp of $\approx 8 \%$ in cells expressing sortilin and TrkA (data not shown), while in FLIM imaging the average lifetime of the sortilin donor fluorophore decreased by $\sim 100-300$ ps when TrkA was present (Fig. 1c). This receptor 
interaction was furthermore demonstrated by surface plasmon resonance (SPR) analysis in which the extracellular domain of sortilin was immobilized on a sensorchip and the soluble Trk ectodomains were present in the fluid phase. All Trks exhibited high affinity binding to sortilin with estimated Kd-values between 10 and $20 \mathrm{nM}$ (Fig. 1d). Because ligand binding to sortilin is conditioned on the release of the receptor propeptide, we tested if Trk, as exemplified by TrkB, can bind to immobilized pro-sortilin (Fig. 1d). We found that prosortilin does not bind Trk, indicating that only the fully processed and mature sortilin is capable of forming heterodimers with Trk. Moreover, binding of all Trk receptors to sortilin was prevented by co-incubation with the soluble sortilin propeptide (data not shown).

To ensure specificity of sortilin-Trk interaction, we also tested if sortilin could bind an unrelated neurotrophin receptor, the glia-derived neurotrophic factor (GDNF) family ligand receptor Ret and one of its ligands neurturin ${ }^{16}$. We failed to detect any interaction of Ret and neurturin with sortilin, neither by co immunoprecipitation (Fig. 1b) nor by SPR analysis (Fig. 1d), suggesting that the binding between sortilin and Trk receptors is unique.

In light of the above, we assessed if endogenous sortilin and Trk can interact in primary neurons. Because both TrkB and sortilin can be detected in hippocampal and cortical neurons, and these cells can be prepared in large quantities, we conducted coimmunoprecipitation experiments of lysates from such cultures. We found that TrkB robustly co-immunoprecipitated with sortilin from hippocampal (Fig. 1e) but also from cortical neurons (data not shown). Furthermore, immunofluorescence staining of nontransfected superior cervical ganglion (SCG) neurons revealed only occasional overlap in the expression pattern of sortilin and TrkA in the cell body (Fig. 1f). Yet, the receptors significantly co-localized in neurites, where they physically interacted as determined by a pFRET efficiency Eapp of 10-15\%. We conclude that sortilin is capable of heterodimerization with Trk receptors in primary neurons of both the central and peripheral nervous system.

\section{Sortilin facilitates anterograde Trk receptor transport}

The heterodimerization of sortilin and Trk in neurites suggested that sortilin might affect transport properties of Trks in neuronal processes. To address this question cultured DRG neurons were transfected with EGFP-TrkA and the movement of vesicles expressing fluorescently labeled TrkA was measured by time-lapse microscopy (Figs. 2a and 2b, Suppl. Movie 1 and 2). Tracking of more than 350 vesicles from both wildtype (wt) and sortilin deficient $\left(\right.$ Sort $\left.^{-/-}\right)$neurons revealed that the ratio of measurable vesicles moving anterograde was reduced by $\sim 4$ fold (from $21.9 \%$ to $4.9 \%$ ) in knockout neurons (Fig. 2c). Contrary, fractions of vesicles moving retrograde or vesicles with no movement were identical in wt and knockout (KO) mice (Fig. 2c). The average vesicle speed in either direction was unaffected by sortilin expression, and amounted to approximately $0.6 \mu \mathrm{m} / \mathrm{s}$ for both genotypes (data not shown).

To examine if sortilin facilitates anterograde Trk transport in peripheral axons in vivo we performed sciatic nerve double ligations. Twenty four hours later, the nerves were removed and the proximal and distal regions probed for receptor expression. Sortilin strongly accumulated proximal (anterograde transport) as well distal (retrograde transport) to the ligature suggesting bi-directional axonal trafficking of the receptor (Fig. 2d). Next we extended these findings by comparing anterograde and retrograde transport of TrkA in the sciatic nerves of Sort $^{+/+}$(wild-type) and Sort $1^{-/-}$mice. Remarkably, the mature $140 \mathrm{kDa}$ form of TrkA was reduced by $\sim 60 \%$ in the proximal region of the nerve in sortilin $\mathrm{KO}$ mice. Contrary, the immature and incompletely glycosylated $110 \mathrm{kDa}$ variant of the protein was unaltered (Figs. 2e and f). Because p75 ${ }^{\mathrm{NTR}}$ trafficking was not affected in these 
experiments, we concluded that sortilin selectively assists in the anterograde transport of mature TrkA.

Prompted by the binding of sortilin and TrkB in cultured hippocampal neurons (Fig. 1e), we finally compared the subcellular distribution of TrkB in the hippocampus by performing membrane fractionation experiments (Fig. 2g). In the wildtype hippocampus, TrkB accumulated in synaptosomes in accordance with previous findings ${ }^{17}$. However, in Sort $1^{-1-}$ mice the amount of TrkB was drastically reduced in these organelles, suggesting that efficient targeting of TrkB to the synapse requires sortilin (Fig. 2h).

Collectively, our data suggested that sortilin is capable of Trk binding in primary neurons and that this interaction is required for efficient anterograde transport and synaptic targeting of Trk receptors.

\section{Impaired MAP kinase activation and neurite outgrowth in cultured neurons from Sort1 ${ }^{-1-}$ mice}

Next we studied the impact of sortilin on TrkA function by investigating NGF signaling in cultured DRG neurons from wild-type and Sort $^{-1-}$ mice. Immunoblotting confirmed that total TrkA levels in these cultures were unaffected by sortilin expression (Suppl. Fig. 1). Addition of $50 \mathrm{ng} / \mathrm{ml} \mathrm{NGF}$ to wildtype neurons for $15 \mathrm{~min}$ induced activation of the MAPkinases ERK1 and -2 (ERK1/2), a well-established downstream target of Trk activation (Fig. $3 a)^{18}$. In contrast, phospho-ERK1/2 was attenuated by approximately $60 \%$ in Sort $^{-1-}$ cultures despite identical levels of total ERK1/2, underscoring the importance of sortilin for efficient TrkA-mediated signaling (Fig. 3b and Suppl. Fig. 1). Since ERK1/2 phosphorylation is a key mediator of neurite outgrowth and differentiation of DRG neurons ${ }^{18}$, we compared NGF-induced sprouting in primary DRG cultures from newborn mice. When cultured in the presence of low NGF concentrations $(25 \mathrm{ng} / \mathrm{ml})$ for $12 \mathrm{hrs}$, knockout neurons exhibited a $\sim 30 \%$ reduction in total neurite length, corroborating the critical role of sortilin for TrkA activity (Fig. $3 \mathrm{c}$-d). Importantly, 10-fold higher NGF concentrations $(250 \mathrm{ng} / \mathrm{ml})$ were able to overcome the impaired sprouting in the Sort $1^{-/-}$ neurons, suggesting that the number of surface exposed mature Trk receptors may be the primary cause of this defect (Fig. 3d).

We subsequently tested if impaired neurite outgrowth of Sort $^{-1-}$ neurons was an intrinsic consequence of sortilin inactivation or whether it was specific for Trk-mediated stimulation. To this end, DRG cultures were stimulated with the neurotrophic factor neurturin that signals through $\operatorname{Ret}^{16}$. As neither Ret nor neurturin physically interact with sortilin (Figs. $1 \mathrm{~b}$ and d), one would predict the Ret/neurturin signaling pathway to be functionally unaffected by sortilin expression. Interestingly, we found no difference in sprouting between wildtype and Sort $^{-1-}$ neurons at 100, 10 or even at $1 \mathrm{ng} / \mathrm{ml}$ neurturin (Fig. 3e), a concentration which is below the previously reported $\mathrm{ED}_{50}$ values of $2-10 \mathrm{ng} / \mathrm{ml}$ for sprouting and survival of primary neuronal cultures ${ }^{19}$.

Taken together our data demonstrated that sortilin selectively impacts on Trk-dependent neuronal differentiation, suggesting that the heterodimerization between sortilin and Trk is biologically meaningful.

\section{Sortilin supports neuronal survival of the peripheral nervous system in vivo}

$\mathrm{p} 75^{\mathrm{NTR}}$ has a dual role in neurotrophin action as it induces apoptosis by proNTs in conjunction with sortilin ${ }^{13}$ but also refines Trk affinity and specificity for their bona fide NTs, thereby augmenting Trk signaling ${ }^{1}$. As a consequence, p75 ${ }^{\mathrm{NTR}}$ knockout $\left(\mathrm{Ngff}^{-/-}\right)$ mice exhibit phenotypes of reduced Trk signaling ${ }^{20}$. To distinguish between the effects of sortilin upon $\mathrm{p} 75^{\mathrm{NTR}}$ versus Trk receptors, we undertook an analysis of double knockout 
mice, reasoning that sortilin-dependent Trk signaling might be unmasked on the $\mathrm{Ngfr}^{-/-}$ background.

In the first series of experiments, we intercrossed sortilin and p $75^{\mathrm{NTR}}$ double heterozygous mice to obtain mice deficient in both loci. Double knockout (DKO) mice were viable and born according to Mendelian ratio (Suppl. Fig. 2). Yet, starting about 4 weeks of age Sort $1^{-1-} / \mathrm{Ngfr}^{-1-}$ mice showed a progressively abnormal waddling gait and an aberrant hindlimb posture (Fig 4a; Suppl. movie 3). Some animals were also noticeable smaller than control littermates (Fig. 4b and Suppl. Fig. 3). The penetrance of this phenotype correlated with the number of knockout alleles, reaching $84 \%$ for the gait phenotype and $37 \%$ for the growth retardation in the DKO (Fig 4b). Because abnormal walking suggested a deficit in the peripheral nervous system, we quantified the number of myelinated fibers in the sciatic nerve as well as the neuronal profiles in the corresponding L4-L5 DRGs of animals 8 weeks of age (Figs. 4c and d). Sort ${ }^{-1-}$ mice did not show any decrease in the amount of myelinated fibers or DRG neurons despite a significant reduction in anterograde trafficking and functional activity (cf. Figs. 2 and 3a-d). Likewise, the number of unmyelinated fibers amounted to $2565+/-240$ and $2712+/-481$ in wild-type and sortilin-deficient animals, respectively (data not shown). However, this observation is in accordance with prior studies in Trk heterozygous mice that have 50\% lower Trk levels, yet an unaltered number of DRG neurons ${ }^{21-23}$. By contrast and in accordance with past findings ${ }^{20,24}$, mice devoid in $\mathrm{p}^{2} 5^{\mathrm{NTR}}$ expression exhibited a $\sim 50 \%$ reduction in DRG neurons, whereas the number of fibers were only lowered by $35 \%$. Interestingly, in the DKO both these values were further decreased by $\sim 35 \%$ relative to $\mathrm{Ngfr}^{-1-}$ mice (Fig. $4 \mathrm{c}$ and d). Our data showed that sortilin deficiency significantly impacts on neuronal survival when $\mathrm{p} 75^{\mathrm{NTR}}$ is absent.

\section{Loss of sortilin expression aggravates TrkA, -B- and -C phenotypes present in p75 ${ }^{\mathrm{NTR}}$ knockout mice}

More than $70 \%$ of DRG neurons express sortilin and almost all p75 ${ }^{\mathrm{NTR}}$ - and Trk-positive neurons contain sortilin (Fig 1a). In agreement with literature, sortilin expression was not confined to a specific neuronal subpopulation within the DRG but present in all subtypes ${ }^{25}$. In detail, sortilin is found in nociceptive neurons including small unmyelinated (peripherin+) non-peptidergic (isolectin IB4+) and peptidergic (calcitonin gene-related peptide (CGRP)+, vanilloid receptor type-1 (TrpV1)+, TrkA+) neurons, and in medium-sized myelinated neurons that stain for neurofilament 200 (NF200) (data not shown). Larger diameter neurons that innervate cutaneous mechanoceptors and convey tactile sensation (TrkB+, NF200+) and large proprioceptive neurons that sense limb movement and position (TrkC+, NF200+) also possessed sortilin (data not shown). Thus, sortilin is expressed in neurons that subserve all perceptual somatic modalities.

Abnormal movement and postures has been described in mice lacking $\operatorname{TrkC}^{26}$ or its ligand neurotrophin-3 (NT3) ${ }^{27}$. Therefore, we compared the number of TrkC/NF200 expressing neurons in $\mathrm{Ngfr}^{-1-}$ and $\mathrm{Sort}^{-1-} / \mathrm{Ngfr}^{-/-}$animals. We found that mice lacking $\mathrm{p} 75^{\mathrm{NTR}}$ exhibited a reduction in NF200- and TrkC-positive cells of approximately 36 and $42 \%$, respectively. In the $\mathrm{Sort}^{-1-} / \mathrm{Ngfi}^{-/-}$these values were further reduced resulting in a total loss of $\sim 60 \%$ of the TrkC-positive neurons (Fig. 5a). As a consequence of the attenuated proprioceptive innervation, muscle spindles were virtually absent in $\mathrm{Sort}^{-1-} / \mathrm{Ngfr}^{-1-}$ mice (Fig. 5b). Collectively, our observations suggest that the profound reduction in TrkC expressing sensory neurons in the Sort $1^{-/-} / \mathrm{Ngfr}^{-/-}$may account for the waddling gait and abnormal hind limb posture (cf. Fig 4a).

We next asked whether other neurotrophin phenotypes might also be affected in the sortilin/ p75 ${ }^{\mathrm{NTR}}$ mutants. Mice heterozygous for BDNF show reduced mechanosensation and TrkBdeficiency is accompanied by loss of Merkel cells and Meissner's corpuscles 28, 28, 29, 29. 
Hence, we subjected Sort $^{-1-} / \mathrm{Ngfi}^{-1-}$ mice to the Von Frey's test, a method to assess mechanical nociception. Application of a force of $1.4 \mathrm{~g}$ induced 80-90\% paw withdrawal response in wild-type and Sort $^{-1-}$ animals but only a $\sim 45 \%$ response in the $\mathrm{p} 75^{\mathrm{NTR}}$ knockouts. However, in Sort $^{-1-} / \mathrm{Ngfr}^{-1-}$ mice this value was reduced to less than $20 \%$ (Fig. $5 c$ ), suggesting an important modulatory role for sortilin in BDNF-dependent mechanosensation. In agreement with this notion, the number of TrkB-positive DRG neurons was decreased by $\sim 20 \%$ in the DKO as compared to p $75^{\mathrm{NTR}}$-null mice (Fig. $5 \mathrm{~d}$ ). This observation showed that even a modest reduction in TrkB-positive neurons dramatically affected the threshold of mechanical pain when the total decline in neurons exceeds $\sim 50 \%$ (Fig. 5d).

TrkA dependent unmyelinated afferents respond to heat-induced noxious stimuli ${ }^{30,31}$. To study if sortilin also affects TrkA dependent thermal algesia we subjected sortilin knockout mice to the Hargreaves test. Paw withdraw latencies were identical in wild-type and Sort $^{-1-}$ animals but increased by $29 \%$ in p $75^{\mathrm{NTR}}$ knockouts in accordance with literature ${ }^{20,24}$ (Fig. 6a). However, an impact of sortilin on nociception was revealed on the $\mathrm{Ngfr}^{-/-}$background inasmuch as the latency was delayed by $72 \%$ in the DKO mice. In accordance with a TrkA phenotype ${ }^{32}$, all subtypes of NGF-dependent nociceptors were significantly reduced in DRG of $\mathrm{Sort}^{-1-} / \mathrm{Ngfr}^{-1-}$ when compared to $\mathrm{Ngfr}^{-/-}$littermates (Fig. 6b). Electron microscopy revealed that unmyelinated nociceptive axons, which are clustered in Remak bundles, had developed normally at postnatal day 15 (P15) $(11.2+/-0.3$ versus $10.1+/-0.5$ neurons per bundle; data not shown) but underwent a dramatic degeneration in the adult (Fig. 6c). As a consequence of this degeneration and the impaired response to noxious stimuli, Sort $1^{-1-}$ $\mathrm{Ngfr}^{-1-}$ mice displayed considerable mutilation or autotomy behavior that resulted in severe injury and deformity of their hindlimb paws after $\sim 3$ months of age ${ }^{33}$ (Fig. 6d). In support of impaired TrkA-mediated signaling in double-deficient mice, we found that NGF-induced ERK1/2 phosphorylation was virtually abolished in DRG cultures from $\mathrm{Sort}^{-1-} / \mathrm{Ngfr}^{-1-}$ mice (Fig. 3a-b).

\section{Heterozygosity for TrkA is embryonic lethal on the sortilin knockout background}

To investigate the specific genetic effects of sortilin upon Trk receptor function, we crossed the sortilin knockouts with mice heterozygous for a mutant TrkA allele $\left(\mathrm{Ntrk}^{+/-}\right)$. Homozygous deficiency for TrkA is embryonic lethal ${ }^{34}$ precluding the generation of DKO. Moreover, both genes are located on chromosome 3 approximately $20 \mathrm{cM}$ apart, requiring allelic cross-over to produce offspring with three knockout alleles (Fig 7a). Progeny from breeding pairs of Sort $^{-1-} / N$ trk $1^{+/+}$and Sort $1^{+/-} / N$ trk $1^{+/-}$mice gave the expected equal frequency of double heterozygous (47\%) and sortilin knockout offspring (40\%), demonstrating that heterozygosity in the TrkA gene locus is not associated with reduced viability. (Fig.7b). Contrary, on the Sort $1^{-1-}$ background, TrkA heterozygosity was accompanied by increased lethality. Thus, Sort $1^{-l-} / N$ trk $1^{+/-}$progeny, as a consequence of allelic cross-over, were born with a frequency of only $2.9 \%$ as compared to $8.6 \%$ for the equivalent Sort $^{+/-} / \mathrm{Ntrk}^{+/+}$genotype. This number corresponds to an embryonic excess lethality of $\sim 65 \%$ for mice harboring three knockout alleles (Fig.7b). At day P27, this value had increased to $89 \%$ which show that Sort $^{-1-} /$ Ntrk $^{+/-}$mice continue to die during the first weeks of life, mimicking the phenotype of the TrkA knockout mouse model ${ }^{34}$.

Because TrkA deficiency is also associated with a severe sympathetic neuropathy ${ }^{34}$, we quantified the superior cervical ganglion (SCG) volume in the animals at P1. Previous studies in Sort $^{-1-}$ did not reveal any obvious signs of impaired TrkA activity in SCG development ${ }^{35}$, However, in accordance with the lethal phenotype, Sort $1^{-1-} / N$ trk $1^{+/-}$mice had significantly smaller SCGs as compared to mice lacking only one TrkA allele (Fig. 7c). 


\section{Discussion}

To assess the full biological functions of sortilin in neurotrophin signaling, we recently generated a sortilin-deficient mouse and confirmed that this receptor is indispensable for proNT-dependent death of neurons during certain stages of development, aging, and brain injury ${ }^{35}$. In this study, we used the same knockout mouse model to show that sortilin also facilitates neurotrophin signaling by escorting Trks along the axonal path to the synapse where receptor activation takes place.

In accordance with our previous findings, we did not observe any overt signs of reduced neurotrophin signaling in Sort $1^{-1-}$ animals $^{35}$. Absence of trophic deficits in vivo is not surprising inasmuch as deficiency for sortilin reduces Trk anterograde transport and activity by approximately $40-50 \%$ (Figs. 2e-h and 3a-d), a value comparable to that present in TrkA, $-\mathrm{B}$, and $-\mathrm{C}$ heterozygous mice that are also phenotypically normal ${ }^{21-23}$. Yet, studies in vitro revealed that cultured DRG neurons isolated from the sortilin knockout mice exhibited $60 \%$ attenuation in NGF-induced ERK phosphorylation and a $\sim 30 \%$ reduction in neurite sprouting (Fig. 3a-d).

We therefore assessed the effects of sortilin on a $\mathrm{Ngff}^{-/-}$or $\mathrm{Ntrk}^{+/-}$background. Sortilin deficiency disclosed an important trophic function of the receptor as these animals presented with severe NT-like deficits. First, Sort $1^{-1-} / \mathrm{Ngfr}^{-/-}$knockouts had an overall reduction in the number of DRG neurons and exhibited a failure to thrive, findings that are compatible with attenuated NT signaling $21-23,34$ (Fig. 4a-b). Second, abnormal hind limb posture, waddling gait and fewer proprioceptive neurons and muscle spindles in the DKOs are also characteristics of mice with disrupted NT-3/TrkC expression ${ }^{26,36}$ (Fig. 5a-b). Third, a reduction in TrkB-positive neurons and impaired tactile sensitivity has also been reported for mice devoid in BDNF/TrkB signaling ${ }^{28,29}$ (Fig. 5c-d). Finally, Sort $1^{-1-} / \mathrm{Ngff}^{-/-}$animals had fewer NGF-dependent nociceptors, suggesting that sortilin is required for full TrkA activity (Fig. 6b). This notion was corroborated in Sort $1^{-1-} / \mathrm{Ntrk}^{+/-}$mice which showed a substantial perinatal lethality and presented with a sympathetic neuropathy reminiscent of that described for TrkA knockout mice ${ }^{34}$ (Fig. 7b-c).

In situ hydridization studies have shown that Trk expression emerges at day E13 and is significant from E15 to birth ${ }^{37}$. Similarly, we previously reported that expression of sortilin commences at E14 in dorsal root ganglia and is maintained through embryonic development ${ }^{38}$. This observation is compatible with a possible role of sortilin in Trk transport during development. However, several lines of evidence suggest the above phenotypes are a consequence of increased degeneration rather than impaired neuronal development. Thus, while motor skills of $\mathrm{Sort}^{-1-} / \mathrm{Ngfr}^{-1-}$ mice appeared normal at birth and during the first weeks of life, the waddling gait debuted at $\sim 4$ weeks of age when development of the peripheral nervous has been completed. Likewise, at P15 the morphology of Remak bundles was normal in DKO mice and the number of non-myelinated neurons indistinguishable from that of the $\mathrm{Ngfr}^{-1-}$ animals (Fig. $6 \mathrm{c}$ and data not shown). However, by $\sim 8$ weeks of age, Remak bundles in $\mathrm{Sort}^{-/-} / \mathrm{Ngfr}^{-/-}$animals had now severely degenerated, whereas axons in the p $75^{\mathrm{NTR}}$ knockout mice remained unaffected (Fig. 6c). Degeneration was accompanied by reduced nociception which, in turn, lead to severe scaring and malformation of the paws in three months old DKO mice (Fig. 6d). Collectively, these findings suggest that sortilin is particularly important for the maintenance of the nervous system when the concentrations of neurotrophins become limited.

A direct interaction between sortilin and the Trk receptors is a plausible explanation for the observed phenotypes. By co-immunoprecipitation and FLIM analysis we found that sortilin physically interacts with TrkA, $-\mathrm{B}$, and $-\mathrm{C}$ in transfected cells (Fig. 1b-c). More importantly, 
we recapitulated this observation with endogenous sortilin and Trk receptors in cultured SCG, and in hippocampal and cortical neurons, supporting an important role for sortilin in Trk trafficking (Fig. 1e-f). Indeed, Sort $1^{-/-}$mice had dramatically impaired anterograde TrkA transport in the sciatic ligation model, and live-imaging of cultured DRG neurons revealed that efficient anterograde, but not retrograde, transport requires expression of sortilin (Fig. 2). In accordance with reduced axonal targeting in the sortilin knockout mice, we found that TrkB was substantially reduced in hippocampal synaptosomal fractions of these animals (Fig. 2h).

Surprisingly, in the sciatic nerve ligation model anterograde transport of the immature and incompletely glycosylated TrkA was independent on sortilin expression ${ }^{15}$ (Fig 2e). This observation was surprising inasmuch both the immature $110 \mathrm{kDa}$ and the mature $140 \mathrm{kDa}$ are capable of sortilin binding as determined by-coimmunoprecipitation (Fig. 1b). Because Trks and sortilin interact via their extracellular domains, this suggests that heterooligomerization between the two receptors is independent of the extent of Trk glycosylation. Interestingly, the inactive precursor of sortilin, pro-sortilin, was unable to bind Trk receptors. The presence of the propeptide prevents premature ligand binding to sortilin in the biosynthetic pathway but furin-mediated cleavage in the TGN conditions the receptor for full biological activity ${ }^{12}$. We therefore propose a model in which vesicles containing the incompletely glycosylated and high-mannose bearing $110 \mathrm{kDa}$ Trk receptor segregates from the Golgi in a compartment that precedes the late TGN where sortilin activation takes place (Fig. 7d). In accordance with such a model, Golgi outposts have been identified in distal dendrites, compatible with local glycosylation and maturation of the $110 \mathrm{kDa}$ Trk receptor variants 39,40 . The biological relevance of having two independent axonal targeting pathways for Trk receptors, of which one only is subject to sortilin-mediated transport, remains to be elucidated.

It has been reported that anterograde Trk transport is mediated by the microtubuledependent motor protein kinesin- $1^{41}$. Kinesin-1, which is one of several members of the kinesin family, is made up by two heavy-chain (KIF5A, -B, or -C) in combination with two light-chain subunits (KLC1, -2 , or -3$)^{2}$. Knockout of KLC-1 is accompanied by reduced anterograde targeting of TrkA but also of other axonally transported proteins including the amyloid precursor protein (APP) and $\beta$-secretase 41,42 . Surprisingly, mice devoid in presenilin-1(PS1) or with overexpression of a PS-1 mutant that continuously mediates $\gamma$ secretase cleavage of APP are also impaired in anterograde transport and surface exposure of TrkB, and in BDNF-induced TrkB autophosphorylation ${ }^{43}$. Although the molecular mechanism remains incompletely understood, it possibly involves dysregulation of the kinesin- 1 motor that targets vesicles that are shared between Trks, APP, $\beta$-secretase, PS- 1 and likely also other membrane proteins ${ }^{41-43}$. Evidence for a direct interaction between Trks and kinesin- 1 comes from a recent study by Arimura and colleagues ${ }^{4}$. They demonstrated that an adaptor complex comprising Slp1/Rab27B/CRMP-2 directly links the cytoplasmic tail of Trk with kinesin-1. However, knockdown of one or more of these adaptors reduced, but did not abrogate, anterograde Trk transport and membrane targeting and signaling. This observation suggests that axonal Trk targeting may be regulated by several trafficking mechanisms, some of which are mediated by either alternative adaptor complexes, distinct kinesins, or various sorting receptors.

What may then be the molecular role of sortilin in Trk trafficking? Several possibilities exist. Sortilin might act as a scaffold receptor that facilitates the formation of a higher order complex between Slp1/Rab27B/CRMP-2, kinesin-1, and the intracellular domain of the Trk receptors. Alternatively, sortilin could itself directly bridge the Trk receptors with the kinesin-1 microtubule motor. So far these questions remains unanswered but it is noteworthy that a yeast-two-hybrid screening has identified KIF1A, a subunit of kinesin-3 that 
transports synaptic vesicles, as a sortilin interaction partner (personal communication: Dr. Peder Madsen, Aarhus University).

In yet another scenario sortilin might act upstream of the conventional anterograde transport machinery to ensure translocation of Trk receptors from the TGN into kinesin-dependent transport vesicles. Several lines of evidence support an essential role of Vps10p-domain receptors in regulating anterograde transport from the late Golgi compartments. While the family member SORLA retains APP in the TGN and impairs its forward transport and plasma membrane insertion, sortilin controls intracellular sorting of BDNF to the regulated secretory pathway ${ }^{6,10}$. Several cytoplasmic adaptor proteins for sortilin have been identified over the past years, but their functional roles have mainly been characterized in terms of endocytosis and Golgi to endosome sorting in non-neuronal cell types. Remarkably, it now appears that one of these adaptors, phosphofurin acidic cluster-sorting protein 1 (PACS-1), which binds to an acidic cluster in the cytosolic domain of sortilin can also mediate anterograde transport in polarized cells ${ }^{6,44}$. Thus, adenoviral expression of a nonphosphorylatable and dominant-negative PACS-1 variant demonstrated that this adaptor is required for trafficking of the olfactory cyclic-nucleotide-gated channel from the cell body to the microtubule based cilia of the apical dendrite in olfactory sensory neurons ${ }^{44}$. Whether PACS-1 is also required for anterograde transport of sortilin is currently under investigation.

A recent study described that axonal targeting of Trk receptors may be accomplished by a more circuitous trafficking pathway than previously appreciated ${ }^{45}$. According to this transcytosis model, Trk receptors are initially embedded into the neuronal soma surface from where they are constitutively endocytosed and axonally targeted via Rab11-positive recycling endosomes to the synapse. Because sortilin is an avidly internalizing receptor, a role for sortilin in transcytosis could be envisioned. However, with the reservation of having used non-polarized cells, we did not observe any reduction in Trk surface expression in 293HEK cells that were stably transfected with sortilin (data not shown). Since constitutive internalization is considered independent on cell polarity, we find it unlikely that sortilin is accountable for the Trk transcytosis.

In conclusion, our data have unraveled sortilin as an important anterograde trafficking receptor for the Trks. We propose a new tripartite model for neurotrophin signaling which we have named the 'neurotrophin triangle' (Fig. 7e): Sortilin is essential for proneurotrophins to form a death signaling complex with $\mathrm{p}^{\mathrm{N}} 5^{\mathrm{NTR} 13,35}$. Signaling by Trks, on the other hand, requires $\mathrm{p} 75^{\mathrm{NTR}}$ expression at the plasma membrane to facilitate binding of mature NTs and to strengthen the trophic signals ${ }^{1}$. To complete this triangular interaction cascade, we have here shown that sortilin supports and fine-tunes neuronal survival by facilitating the anterograde transport of Trk receptors and securing their proper exposure in the synapse.

\section{Methods}

Animals

Sort $1^{-/-}, \mathrm{Ngfr}^{-/-}$, and $\mathrm{Ntrk}^{-1-}$ mice have been described previously ${ }^{20,34,35}$. DKO were generated by breeding of double heterozygous animals and littermates served as controls. Results obtained on a pure C57BL/6J genetic background and on hybrid 129SvEmcTer X C57BL/6J and 129SvEmcTer X BALB/cJ lines were similar.

\section{Neuron cultures}

DRG and SCG cultures were prepared from P0-P3 mice by digesting the isolated ganglia in trypsin $(0.125 \%)$ and collagenase $(1 \mathrm{mg} / \mathrm{ml})$, followed by seeding on PLL/laminin in DMEM supplemented with 10\% FCS, 1mM glutamine, Primocin (Amaxa), $20 \mu \mathrm{M}$ 5- 
Fluoro-2'-deoxyuridine, $20 \mu \mathrm{M}$ Uridine and $2 \mathrm{nM}$ NGF. Hippocampus neurons were prepared from P0 mice by digestion with papain ( 20 units $/ \mathrm{ml}$ ) for $30 \mathrm{~min}$, followed by seeding on PLL/laminin in neurobasal supplemented with B27, glutamax and primocin.

\section{FRET/FLIM}

Dissociated SCG neurons as well as HEK293 cells stably expressing sortilin or sortilin $+\operatorname{TrkA}^{13}$ were incubated with goat anti-sortilin (R\&D) and rabbit anti-Trk (RTA, L. Reichardt, UCSF) followed by Alexa-conjugated donkey secondary antibodies (anti-goat Alexa488 and anti-rabbit Alexa 568). Samples were analyzed on a Zeiss confocal LSM 510 META microscope using a 40× NA 1.2 C-Apochromat (HEK293) or 63× NA 1.4 PlanApochromat objective (SCG). FRET: Sensitized acceptor emission FRET were performed as described ${ }^{46}$. Briefly, Donor and acceptor signals were detected through 500-530 nm and $565-615 \mathrm{~nm}$ emission filters following $488 \mathrm{~nm}$ (donor) or 543nm (acceptor) excitation, respectively. The collected FRET images were analyzed by ImageJ-based PFRET software, enabling us to correct for donor- and acceptor spectral bleedthroughs (DSBT and ASBT) on donor- and acceptor signal levels. All calculations/corrections were performed on background-subtracted images. Lower bounds for signal levels used in ASBT and DSBT correction calculations were set to 25 . ROI's were chosen to be $5 \times 5$ and only ROI signal levels $\geq 20$ for all pixels in the ROI's were included for analysis. Measurements were performed on 30 HEK293 cells and 10 SCG neurons. FLIM: The microscope was equipped with a mode-locked Ti-sapphire laser (Mai-Tai Broadband, Spectra Physics), photon counting card (SPC830), detector and software from Becker and Hickl (SPCM v. 2.9.4.1993). Alexa488 was two-photon excited with $20 \mathrm{~mW}$ at $760 \mathrm{~nm}$ and the fluorescence detected after reflection off a 535DCXR dichroic (Chroma) and transmission through ET505/40m-2p and E700SP-2p filters (Chroma). Fluorescence decay curves measured for Alexa488 (Sortilin) in the presence and absence of Alexa568 (TrkA) were fitted to monoexponential decay functions in SPCImage, using bin 1, amplitude threshold 50, scatter parameter fixed to 0 , leaving all other parameters free. The instrument response function was calculated automatically by SPCImage. Histograms with lifetime as well as Chi-square value distributions obtained from the fitted pixels were generated by the software, depicted as pixel intensity weighted, and the mean lifetime and mean Chi-square value of each distribution was evaluated for each data file. Measurements were performed on 16 HEK293 cells.

\section{MAPK activation assay}

10-12 DIV DRG cultures were washed $4 \times 1 \mathrm{~h}$ in $37^{\circ} \mathrm{C}$ DMEM followed by incubation for 15 min at $37^{\circ} \mathrm{C}$ with DMEM $+2 \mathrm{nM}$ NGF. The cells lysate (lysis buffer: $20 \mathrm{mM}$ Tris $\mathrm{pH} 8,1 \%$ NP40, 10mM EDTA, complete protease inhibitor cocktail (Roche), $2 \mathrm{mM}$ sodium orthovanadate and phosphatase inhibitor cocktail 1 (Sigma)) was subjected to SDS-PAGE (equal amounts of protein loaded), blotted, probed with anti-phospho-p42/44 MAP kinase (P-MAPK) antibody (Cell Signaling) and HRP-conjugated secondary antibody (Dako) and visualized with ECL substrate (Pierce). Equal loading was verified by blotting against betaactin. Densitometry analysis of bands was performed using Multi Gauge 3.0 software (FujiFilm Life Science). NGF stimulated increase in P-MAPK was normalized to control within each experiment $(n=3)$.

\section{Neurite outgrowth}

DRG neurons from P0-P1 mice were incubated with NGF (1 or $10 \mathrm{nM})$ or Neuturin $(1,10$ or $100 \mathrm{ng} / \mathrm{ml}$ ) for $12 \mathrm{~h}$, fixed and incubated with TUJ-1 primary antibody (Chemicon) followed by Alexa488-conjugated secondary antibody (Molecular probes). Images of complete branches were quantified by NeuriteTracer plugin for ImageJ software (>20 neurons per 
experiment for each genotype). Average total length per neuron was calculated and the mean value for each experiment normalized to wild-type ( $n=4$ to 6 )

\section{Live cell imaging}

P0 DRG neurons were transfected (Amaxa) with EGFP-TrkA and seeded in MatTek glassbottom chamber. The following day the cells were depleted of NGF for $6 \mathrm{~h}$ and placed in a $\mathrm{CO}_{2}$ /temperature incubator attached to the microscope. Movies were recorded on a Zeiss LSM510 63x/1.4 oil objective and 30 frames per minute setting. A total of 350 vesicles were analyzed for each genotype in 8-10 neurons prepared in 3 independent experiments. Vesicle movement were analyzed with ImageJ using the KymoToolBox plugin (kindly provided by Fabrice Cordelières, Université Paris-Sud Orsay - France).

\section{Surface Plasmon Resonance}

SPR analysis were performed on a Biacore ${ }^{\circledR} 3000$ as previously described ${ }^{13}$. Briefly, soluble sortilin was immobilized (at $10-15 \mu \mathrm{g} / \mathrm{ml}$ ) on a CM5 chip and remaining coupling sites were blocked with $1 \mathrm{M}$ ethanolamine. Sample and running buffer was $10 \mathrm{mM}$ HEPES, $150 \mathrm{mM}$ $\left(\mathrm{NH}_{4}\right)_{2} \mathrm{SO}_{4}, 1.5 \mathrm{mM} \mathrm{CaCl}_{2}, 1 \mathrm{mM}$ EGTA, $0.005 \%$ Tween-20 pH 7.4. Fc-fusions proteins of p75 ${ }^{\mathrm{NTR}}$, RET, TrkA, -B and -C (R\&D Biotechnology) were applied at 20, 100 and $500 \mathrm{nM}$ in increasing concentration, and the sensor chip regenerated in a $10 \mathrm{mM}$ glycine-HCl buffer after each analytic cycle. The SPR signal was expressed in relative response units (RU) as the response obtained in a control flow channel was subtracted.

\section{Immunoprecipitation}

HEK293 cell lines stably expressing combinations of sortilin and TrkA or C or RET as well as dissociated hippocampus and cortical cultures were treated with DSP crosslinker (Pierce Biotechnology), lysed in TNE lysis buffer (20 mM Tris pH 8, 1\% NP40, 10mM EDTA, complete protease inhibitor cocktail) and the cell lysate immunopreciptated with anti-Trk antibody (C14, Santa Cruz Biotechnology) or polyclonal anti-sortilin antibody linked to sepharose $\mathrm{G}$ beads (Amersham Biosciences). For neuronal cultures, control IP was performed with rabbit pre-immune serum. Following elution, SDS-PAGE and blotting, the proteins were probed with anti-sortilin (BD Transduction Labs), anti-Trk (C14, Santa Cruz Biotechnology), anti-TrkB or anti-Ret (R\&D Systems) and visualized with HRP-conjugated secondary antibody (Dako) and ECL substrate (Pierce).

\section{Hippocampus subcellular fractionation}

The procedure for preparation of hippocampal synaptosomes was performed essentially as described by Blackstone et $\mathrm{al}^{47}$. In brief, hippocampus was isolated from $12 \mathrm{wt}$ or Sort $^{-1-}$ mice (12-16 weeks old) and homogenized in 0.32 sucrose, 4 mM Hepes, $\mathrm{pH} 7.4$ containing proteinase inhibitors. For each experiment, wt and Sort $^{-1-}$ samples were processed in parallel in order to directly compare fractions $(n=4)$. P1 and $S 1$ are the pellet and supernatant, respectively, after centrifugation for $10 \mathrm{~min}$ at $1,000 \times \mathrm{g}$. S1 was further centrifuged for $15 \mathrm{~min}$ at $10,000 \times \mathrm{g}$ to obtain supernatant S2 (light membranes) and the pellet P2, a crude synaptosomal preparation. Solubilized P2 was centrifuged for $15 \mathrm{~min}$ at $10,000 \times \mathrm{g}$ and the resulting pellet was solubilized in ice cold $\mathrm{H}_{2} \mathrm{O}$ using a glass-teflon homogenizer and centrifuged again at 25,000 $\times \mathrm{g}$ for 20 min generating the synaptosomal membrane fraction $\mathrm{P} 3$ and a supernatant $\mathrm{S} 3$ enriched in presynaptic vesicles. All centrifugation steps were performed at $4^{\circ} \mathrm{C}$. Total protein concentration in fractions was determined using the Bicinchoninic Acid kit from Sigma and equal amounts of proteins of each fraction was separated by reducing SDS-PAGE and analyzed by Western blotting for the presence of TrkB and synaptophysin. 


\section{Immunohistochemistry}

L4/L5 DRG was fixed in 4\% PFA, cryoprotected in 25\% sucrose, embedded in TissueTek $®$ and cut $(14 \mu \mathrm{m})$ on a cryostat, thaw-mounted on Superfrost Plus slides and kept at $-80^{\circ} \mathrm{C}$ until use. After antigen retrieval $\left(0.05 \%\right.$ tween 20 in $10 \mathrm{mM}$ citrate buffer, $\mathrm{pH} 6.0$ at $95^{\circ} \mathrm{C}$ for $20 \mathrm{~min}$ ) and blocking (5\% goat serum $+0.3 \%$ TritonX100) the tissue was incubated with antibodies against peripherin (Abcam), heavy neurofilament 200 (Abcam), TrpV1 (Neuromics), TrkA (L. Reichardt, UCSF), TrkB (Santa Cruz), TrkC (R\&D), CGRP (Biomol), p $75^{\mathrm{NTR}}(9651)^{48}$ or sortilin (Alomone labs). Alexa-conjugated secondary antibodies were obtained from Molecular Probes Inc. IB4-reactive neurons were visualized by IB4-FITC (Sigma-Aldrich L2895, $10 \mu \mathrm{g} / \mathrm{ml}$ ). Images were recorded on a Zeiss LSM510 confocal microscope. For neuron profile estimation, every $3^{\text {rd }}$ section was stained with cresyl violet and the total number of neuronal profiles with nucleoli counted in L4 and L5 DRG. Method validity was verified by several control experiments, e.g. comparing p75 $5^{\mathrm{NTR}}-/-$ counts from adult mice with published data obtained by the design-based optical fractionator principle, yielding identical results $(47.2+/-3.0 \%$ versus $47.9+/-7.7 \%$ of wt, respectively). Quantification of neurons positive for molecular markers was achieved by counting positive and total neuron profiles on at least 20 independent (non-adjacent) sections (more than 10 DRGs per genotype evaluated in total). Marker distributions for the wt were performed as described $24,49-53$.

\section{Histology of sciatic nerve}

N. Ischiadicus was dissected from adult mice, fixed in 4\% PFA, incubated in $1 \%$ osmiumtetroxide in cacodylate buffer, dehydrated in increasing ethanol gradient (30\%-99\%), incubated in Technovit $7100+$ Hardener I and finally embedded in Technovit $7100+$ Hardener II (Kulzer, Germany). Tissue blocks were sectioned $(3 \mu \mathrm{m})$, stained with Toluidin Blue (TB) and number of myelinated axons counted.

\section{Electron microscopy}

The sciatic nerve was dissected and processed as described previously ${ }^{54}$. Briefly, tissue from transcardially perfusion fixed mice (4\% PFA) was postfixed in 4\% PFA+2.5\% glutaraldehyde. Following treatment with $1 \%$ osmium tetroxide they were dehydrated in a graded ethanol series and propylene oxide and embedded in Poly/BedR 812 (Polysciences, Inc., Eppelheim, Germany). Semithin sections were stained with toluidine blue. Ultrathin sections $(70 \mathrm{~nm})$ were contrasted with uranyl acetate and lead citrate and examined with a Zeiss 910 electron microscope. Digital images were taken with a high-speed slow scan CCD camera (Proscan) at an original magnification of $1250 \times$ and 5000x.

\section{Sciatic nerve ligation}

Adult mice were anaesthetized with ketamine/xylazine and the right sciatic nerve was double ligated at mid-high level. After $24 \mathrm{~h}$ the animals were sacrificed and 3-4mm sciatic nerve proximal and distal to the ligation (or approximately at the same location for control side) was isolated. Nerve segments from 8-10 animals of each genotype were pooled for each experiment ( 3 independent experiments) and homogenized/lysed in lysis buffer (as for MAPK activation assay). 100 $\mu$ g protein per lane was separated by SDS-PAGE and western blotting, followed by incubation with anti-TrkA, anti-p $75^{\text {NTR }}$ or anti-sortilin (antibodies as described above) followed by HRP-conjugated secondary antibody. Densitometry analysis was performed using Multi Gauge 3.0 software and band intensity normalized to wt for each western blot. 


\section{Behavioral tests}

Animals were kept under a $12 \mathrm{~h} / 12 \mathrm{~h}$ day/might cycle and had unrestricted access to water and food. On the day of the experiment unrestrained animals were allowed to habituate for 1 hour before start of the experiments.

Hargreaves test-The radiant heat source (37380, Ugo Basile) was calibrated using a Heat Flow I.R, Radiometer (37300, Ugo Basile) and kept at 50\% $\left(190 \mathrm{~mW} / \mathrm{cm}^{2}\right)$ in all tests. The hind paws were tested alternately with $>5$ minutes between consecutive tests, and 3-5 measurements were obtained for each side. 10-14 animals were tested.

Von Frey test-Von Frey hairs of increasing bending force (calibrated $0.008 \mathrm{~g}-1.4 \mathrm{~g}$ from Touch-Test ${ }^{\circledR}$ Sensory Evaluators, North Coast Medical, CA) were each applied 5 times to both sides of the plantar hind limb area from below through the mesh floor, and the number of withdrawals counted.

\section{SCG volume}

SCG ganglia from P28 mice fixed in 4\% PFA and cryo-sectioned $(20 \mu \mathrm{m})$ were incubated with anti-tyrosine hydroxylase antibody (Pel Freeze), followed by biotinylated anti-rabbitIgG (Amersham) and Streptavidin-HRP-conjugated (DakoCytomation), visualized by DAB and the samples dehydrated and mounted in Eukitt. SCG neurons were photographed (Leica LM50) and the individual volume estimated by the principle of Cavalieri as described previously ${ }^{55}$.

\section{Muscle spindle}

The lower hind legs of neonatal mice were snap frozen in isopentane and cryosectioned transversely at $10-12 \mu \mathrm{m}$. Every $6^{\text {th }}$ section were incubated with antibody S46 (DSHB) as a marker for slow tonic myosin heavy chain (MHCst) intrafusal fibers located in muscle spindles ${ }^{56}$. The tissue were preincubated with goat anti-mouse Fab fragments (Jackson labs) to reduce unspecific binding of the secondary antibody, followed by HRP-conjugated rabbit anti-mouse (Dako) and visualized with DAP. Qualitative estimation of spindle numbers and size were obtained upon examination using an $\mathrm{x} 4$ objective (Leica microscope).

\section{Statistical analysis}

Statistical significance was determined by 2-tailed student's t-test or ANOVA after testing for normal distribution when appropriate. One-sample tests were applied to quantification from DRG neurite outgrowth, MAPK phosphorylation assay as well as sciatic TrkA transport. Breeding outcome was evaluated by Chi-square test.

\section{Supplementary Material}

Refer to Web version on PubMed Central for supplementary material.

\section{Acknowledgments}

This work was supported by the Lundbeck Foundation, The Danish Medical Research Council, Elvira and Rasmus Rissforts Foundation, MEMORIES (EU, Framework Programme 6), NIH (NS21072 and AG025970), the DFG, NeuronIcon, Danish Council for Strategic Research, Center for Stochastic Geometry, and advanced Bioimaging supported by the Villum Foundation. 


\section{Reference List}

1. Chao MV. Neurotrophins and their receptors: a convergence point for many signalling pathways. Nat. Rev. Neurosci. 2003; 4:299-309. [PubMed: 12671646]

2. Hirokawa N, Noda Y, Tanaka Y, Niwa S. Kinesin superfamily motor proteins and intracellular transport. Nat. Rev. Mol. Cell Biol. 2009; 10:682-696. [PubMed: 19773780]

3. Hirokawa N, Takemura R. Molecular motors and mechanisms of directional transport in neurons. Nat. Rev. Neurosci. 2005; 6:201-214. [PubMed: 15711600]

4. Arimura N, et al. Anterograde transport of TrkB in axons is mediated by direct interaction with Slp1 and Rab27. Dev. Cell. 2009; 16:675-686. [PubMed: 19460344]

5. Arimura N, et al. Anterograde transport of TrkB in axons is mediated by direct interaction with Slp1 and Rab27. Dev. Cell. 2009; 16:675-686. [PubMed: 19460344]

6. Willnow TE, Petersen CM, Nykjaer A. VPS10P-domain receptors - regulators of neuronal viability and function. Nat. Rev. Neurosci. 2008; 9:899-909. [PubMed: 19002190]

7. Nielsen MS, et al. The sortilin cytoplasmic tail conveys Golgi-endosome transport and binds the VHS domain of the GGA2 sorting protein. EMBO J. 2001; 20:2180-2190. [PubMed: 11331584]

8. Petersen CM, et al. Molecular identification of a novel candidate sorting receptor purified from human brain by receptor-associated protein affinity chromatography. J. Biol. Chem. 1997; 272:3599-3605. [PubMed: 9013611]

9. Sarret $\mathrm{P}$, et al. Distribution of NTS3 receptor/sortilin mRNA and protein in the rat central nervous system. J. Comp Neurol. 2003; 461:483-505. [PubMed: 12746864]

10. Chen ZY, et al. Sortilin controls intracellular sorting of brain-derived neurotrophic factor to the regulated secretory pathway. J. Neurosci. 2005; 25:6156-6166. [PubMed: 15987945]

11. Quistgaard EM, et al. Ligands bind to Sortilin in the tunnel of a ten-bladed beta-propeller domain. Nat. Struct. Mol. Biol. 2009; 16:96-98. [PubMed: 19122660]

12. Munck PC, et al. Propeptide cleavage conditions sortilin/neurotensin receptor-3 for ligand binding. EMBO J. 1999; 18:595-604. [PubMed: 9927419]

13. Nykjaer A, et al. Sortilin is essential for proNGF-induced neuronal cell death. Nature. 2004; 427:843-848. [PubMed: 14985763]

14. Teng HK, et al. ProBDNF induces neuronal apoptosis via activation of a receptor complex of p75NTR and sortilin. J. Neurosci. 2005; 25:5455-5463. [PubMed: 15930396]

15. Martin-Zanca D, Oskam R, Mitra G, Copeland T, Barbacid M. Molecular and biochemical characterization of the human trk proto-oncogene. Mol. Cell Biol. 1989; 9:24-33. [PubMed: 2927393]

16. Runeberg-Roos P, Saarma M. Neurotrophic factor receptor RET: structure, cell biology, and inherited diseases. Ann. Med. 2007; 39:572-580. [PubMed: 17934909]

17. Gomes RA, Hampton C, El-Sabeawy F, Sabo SL, McAllister AK. The dynamic distribution of TrkB receptors before, during, and after synapse formation between cortical neurons. J. Neurosci. 2006; 26:11487-11500. [PubMed: 17079678]

18. Reichardt LF. Neurotrophin-regulated signalling pathways. Philos. Trans. R. Soc. Lond B Biol. Sci. 2006; 361:1545-1564. [PubMed: 16939974]

19. Thang SH, Kobayashi M, Matsuoka I. Regulation of glial cell line-derived neurotrophic factor responsiveness in developing rat sympathetic neurons by retinoic acid and bone morphogenetic protein-2. J. Neurosci. 2000; 20:2917-2925. [PubMed: 10751444]

20. Lee KF, et al. Targeted mutation of the gene encoding the low affinity NGF receptor p75 leads to deficits in the peripheral sensory nervous system. Cell. 1992; 69:737-749. [PubMed: 1317267]

21. Ernfors P, Lee KF, Kucera J, Jaenisch R. Lack of neurotrophin-3 leads to deficiencies in the peripheral nervous system and loss of limb proprioceptive afferents. Cell. 1994; 77:503-512. [PubMed: 7514502]

22. Klein R, et al. Targeted disruption of the trkB neurotrophin receptor gene results in nervous system lesions and neonatal death. Cell. 1993; 75:113-122. [PubMed: 8402890]

23. Minichiello L, et al. Differential effects of combined trk receptor mutations on dorsal root ganglion and inner ear sensory neurons. Development. 1995; 121:4067-4075. [PubMed: 8575307] 
24. Bergmann I, et al. Analysis of cutaneous sensory neurons in transgenic mice lacking the low affinity neurotrophin receptor p75. Eur. J. Neurosci. 1997; 9:18-28. [PubMed: 9042565]

25. Arnett MG, Ryals JM, Wright DE. Pro-NGF, sortilin, and p75NTR: potential mediators of injuryinduced apoptosis in the mouse dorsal root ganglion. Brain Res. 2007; 1183:32-42. [PubMed: 17964555]

26. Klein R, et al. Disruption of the neurotrophin-3 receptor gene trkC eliminates la muscle afferents and results in abnormal movements. Nature. 1994; 368:249-251. [PubMed: 8145824]

27. Farinas I, Jones KR, Backus C, Wang XY, Reichardt LF. Severe sensory and sympathetic deficits in mice lacking neurotrophin-3. Nature. 1994; 369:658-661. [PubMed: 8208292]

28. Carroll P, Lewin GR, Koltzenburg M, Toyka KV, Thoenen H. A role for BDNF in mechanosensation. Nat. Neurosci. 1998; 1:42-46. [PubMed: 10195107]

29. Perez-Pinera $P$, et al. Characterization of sensory deficits in TrkB knockout mice. Neurosci. Lett. 2008; 433:43-47. [PubMed: 18248898]

30. Chao MV, Rajagopal R, Lee FS. Neurotrophin signalling in health and disease. Clin. Sci. (Lond). 2006; 110:167-173. [PubMed: 16411893]

31. Chuang $\mathrm{HH}$, et al. Bradykinin and nerve growth factor release the capsaicin receptor from PtdIns(4,5)P2-mediated inhibition. Nature. 2001; 411:957-962. [PubMed: 11418861]

32. Silos-Santiago I, et al. Non-TrkA-expressing small DRG neurons are lost in TrkA deficient mice. J. Neurosci. 1995; 15:5929-5942. [PubMed: 7666178]

33. Coderre TJ, Grimes RW, Melzack R. Deafferentation and chronic pain in animals: an evaluation of evidence suggesting autotomy is related to pain. Pain. 1986; 26:61-84. [PubMed: 3526256]

34. Smeyne RJ, et al. Severe sensory and sympathetic neuropathies in mice carrying a disrupted Trk/ NGF receptor gene. Nature. 1994; 368:246-249. [PubMed: 8145823]

35. Jansen $\mathrm{P}$, et al. Roles for the pro-neurotrophin receptor sortilin in neuronal development, aging and brain injury. Nat. Neurosci. 2007; 10:1449-1457. [PubMed: 17934455]

36. Tessarollo L, Vogel KS, Palko ME, Reid SW, Parada LF. Targeted mutation in the neurotrophin-3 gene results in loss of muscle sensory neurons. Proc. Natl. Acad. Sci. U. S. A. 1994; 91:1184411848. [PubMed: 7991545]

37. Mu X, Silos-Santiago I, Carroll SL, Snider WD. Neurotrophin receptor genes are expressed in distinct patterns in developing dorsal root ganglia. J. Neurosci. 1993; 13:4029-4041. [PubMed: 8366358]

38. Hermans-Borgmeyer I, Hermey G, Nykjaer A, Schaller C. Expression of the 100-kDa neurotensin receptor sortilin during mouse embryonal development. Brain Res. Mol. Brain Res. 1999; 65:216219. [PubMed: 10064893]

39. Horton AC, Ehlers MD. Neuronal polarity and trafficking. Neuron. 2003; 40:277-295. [PubMed: 14556709]

40. Merianda TT, et al. A functional equivalent of endoplasmic reticulum and Golgi in axons for secretion of locally synthesized proteins. Mol. Cell Neurosci. 2009; 40:128-142. [PubMed: 19022387]

41. Kamal A, Almenar-Queralt A, LeBlanc JF, Roberts EA, Goldstein LS. Kinesin-mediated axonal transport of a membrane compartment containing beta-secretase and presenilin-1 requires APP. Nature. 2001; 414:643-648. [PubMed: 11740561]

42. Hirokawa N, Takemura R. Molecular motors and mechanisms of directional transport in neurons. Nat. Rev. Neurosci. 2005; 6:201-214. [PubMed: 15711600]

43. Lazarov $\mathrm{O}$, et al. Impairments in fast axonal transport and motor neuron deficits in transgenic mice expressing familial Alzheimer's disease-linked mutant presenilin 1. J. Neurosci. 2007; 27:70117020. [PubMed: 17596450]

44. Jenkins PM, Zhang L, Thomas G, Martens JR. PACS-1 mediates phosphorylation-dependent ciliary trafficking of the cyclic-nucleotide-gated channel in olfactory sensory neurons. J. Neurosci. 2009; 29:10541-10551. [PubMed: 19710307]

45. Ascano M, Richmond A, Borden P, Kuruvilla R. Axonal targeting of Trk receptors via transcytosis regulates sensitivity to neurotrophin responses. J. Neurosci. 2009; 29:11674-11685. [PubMed: 19759314] 
46. Wallrabe H, Elangovan M, Burchard A, Periasamy A, Barroso M. Confocal FRET microscopy to measure clustering of ligand-receptor complexes in endocytic membranes. Biophys. J. 2003; 85:559-571. [PubMed: 12829510]

47. Blackstone $\mathrm{CD}$, et al. Biochemical characterization and localization of a non-N-methyl-D-aspartate glutamate receptor in rat brain. J. Neurochem. 1992; 58:1118-1126. [PubMed: 1371146]

48. Huber LJ, Chao MV. Mesenchymal and neuronal cell expression of the p75 neurotrophin receptor gene occur by different mechanisms. Dev. Biol. 1995; 167:227-238. [PubMed: 7851645]

49. Chen CL, et al. Runx 1 determines nociceptive sensory neuron phenotype and is required for thermal and neuropathic pain. Neuron. 2006; 49:365-377. [PubMed: 16446141]

50. Gjerstad MD, Tandrup T, Koltzenburg M, Jakobsen J. Predominant neuronal B-cell loss in L5 DRG of p75 receptor-deficient mice. J. Anat. 2002; 200:81-87. [PubMed: 11833656]

51. Holmes FE, et al. Targeted disruption of the galanin gene reduces the number of sensory neurons and their regenerative capacity. Proc. Natl. Acad. Sci. U. S. A. 2000; 97:11563-11568. [PubMed: 11016970]

52. Negri L, et al. Impaired nociception and inflammatory pain sensation in mice lacking the prokineticin receptor PKR1: focus on interaction between PKR1 and the capsaicin receptor TRPV1 in pain behavior. J. Neurosci. 2006; 26:6716-6727. [PubMed: 16793879]

53. Zwick M, et al. Glial cell line-derived neurotrophic factor is a survival factor for isolectin B4positive, but not vanilloid receptor 1-positive, neurons in the mouse. J. Neurosci. 2002; 22:40574065. [PubMed: 12019325]

54. Wetzel C, et al. A stomatin-domain protein essential for touch sensation in the mouse. Nature. 2007; 445:206-209. [PubMed: 17167420]

55. Jansen $\mathrm{P}$, et al. Roles for the pro-neurotrophin receptor sortilin in neuronal development, aging and brain injury. Nat. Neurosci. 2007; 10:1449-1457. [PubMed: 17934455]

56. Sokoloff AJ, Li H, Burkholder TJ. Limited expression of slow tonic myosin heavy chain in human cranial muscles. Muscle Nerve. 2007; 36:183-189. [PubMed: 17486578] 
a

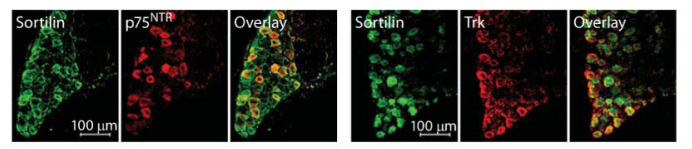

b
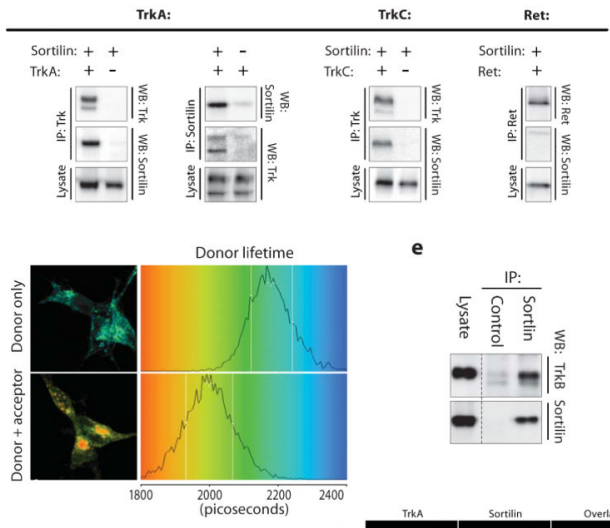

e

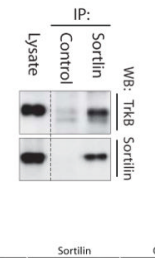

d
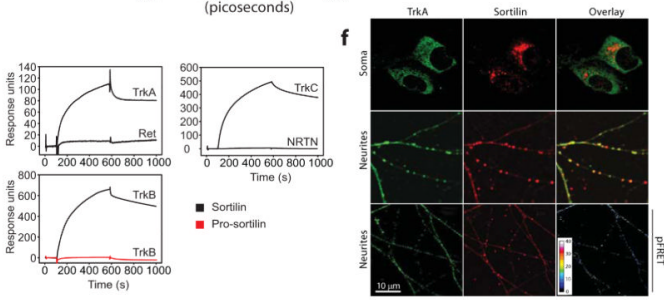

Figure 1.

Sortilin interacts with Trk receptors. a, Co-localization of sortilin with $\mathrm{p} 75^{\mathrm{NTR}}$ and Trk receptors in DRG neurons. b, Left: co-immunoprecipitation of sortilin and TrkA in HEK293 using pan-anti-Trk or anti-sortilin antibody, respectively (IP), Middle: coimmunoprecipitation of sortilin with TrkC in HEK293 using pan-anti-Trk (IP), Right: No co-immunoprecipitation of sortilin with Ret. Proteins are visualized by western blotting (WB). c, Interaction between sortilin and TrkA in HEK293 cells as measured by reduction in donor fluorophore Alexa488 (sortilin) lifetime (FLIM) in the presence of the acceptor fluorophore Alexa568 (TrkA). d, Surface plasmon resonance analysis of receptor interactions; binding of extracellular domains of TrkA, $-\mathrm{B}$ and $-\mathrm{C}$ to the immobilized sortilin ectodomain. Absent binding of sortilin to Ret and neurturin (NRTN) served as negative controls. Lower panel: No TrkB binding to immobilized prosortilin (red curve) e, co-immunoprecipitation of TrkB from hippocampal neurons using anti-sortilin antibody but not by pre-immune IgG. f, Immunofluorescence staining of endogenous sortilin and TrkA in cultured SCG neurons. pFRET signals of co-localized receptors was obtained in neurites (bottom right). 


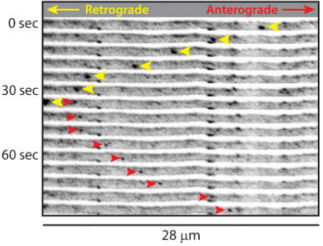

b

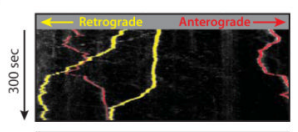

$44 \mu \mathrm{m}$

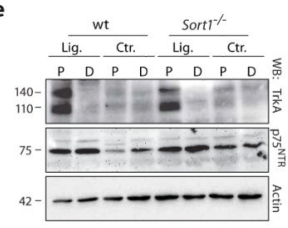

g

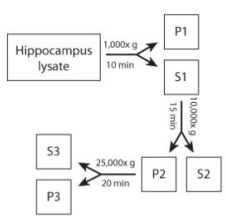

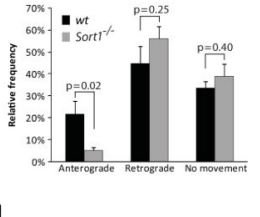

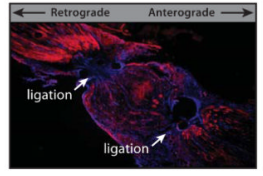

f

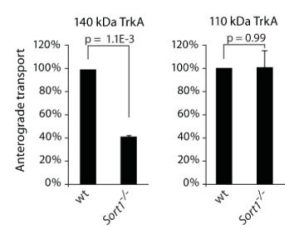

h

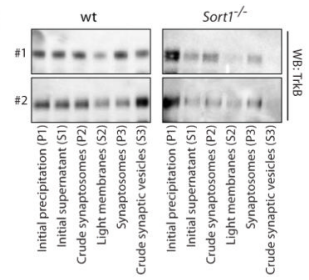

Figure 2.

Endogenous sortilin facilitates TrkA trafficking and signaling in neurons. a, Movement of EGFP-TrkA in neurites of cultured DRG neurons. Arrows indicate anterograde (red) and retrograde (yellow) movement of vesicles. b. Kymograph of a neurite as shown in panel a. Images were captured every $2 \mathrm{~s}$ for $300 \mathrm{~s}$ per neurite and kinetic parameters for each vesicle trace obtained by ImageJ KymoToolBox plugin analysis. c, Relative frequencies of anterograde, retrograde or no movement of approximately 350 vesicles for each genotype analyzed as described in b. d, Immunofluorescence of sortilin (red) and DAPI staining (blue) of a double ligated rat sciatic nerve. Accumulation of sortilin proximal and distal to the ligation illustrates anterograde and retrograde sortilin transport processes, respectively. No sortilin accumulation was observed in sham operated mice (data not shown). e, WB of double ligated sciatic nerves in wild-type and sortilin-deficient mice; anterograde TrkA transport (top), bidirectional transport of $\mathrm{p} 75^{\mathrm{NTR}}$ (middle) and actin loading control (bottom) based on accumulation of immunoreactive protein bands proximal (P) and/or distal (D) to the ligature. $\mathbf{f}$, Quantification of the accumulation of mature and immature TrkA in sortilin knockouts relative to wild-type mice ( $\mathrm{n}=3$ ), cf. panel e. $\mathbf{g}, \mathbf{h}$, Hippocampal membrane fractionation. Schematic overview of sample preparation (panel $g$ ). Western blot analysis of TrkB in two representative experiments (\#1 and \#2) illustrates altered subcellular distribution of TrkB in the Sort $^{-/-}$hippocampus (panel $h$ ) $(\mathrm{n}=4)$. 
a

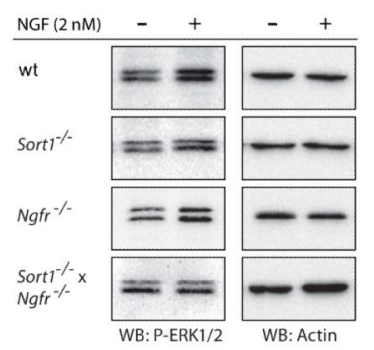

c

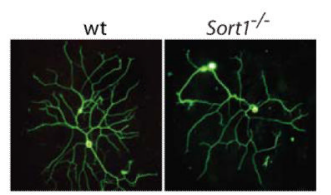

e

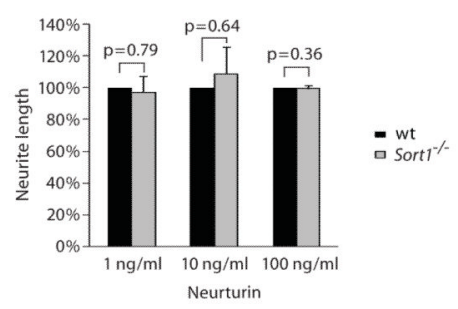

b

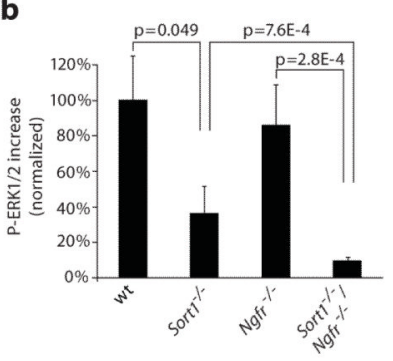

d

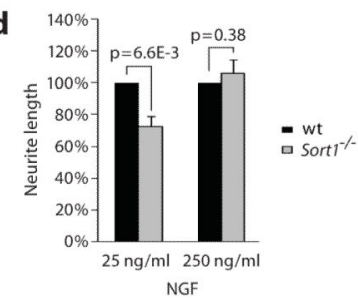

Figure 3.

Sortilin facilitates Trk signaling in neurons. a, WB of phospho-ERK1/2 (MAPK) in primary DRG neuron cultures after stimulation with NGF for $10 \mathrm{~min}$. b, Quantification of ERK1/2 activation from 5 experiments as depicted in panel a. c, TUJ-1 staining of DRG neurons $12 \mathrm{~h}$ after plating. d, e, Quantification of neurite length $(n=4)$ after stimulation with NGF (panel d) or the Ret ligand neurturin (panel e). 
a

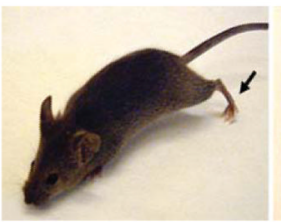

C

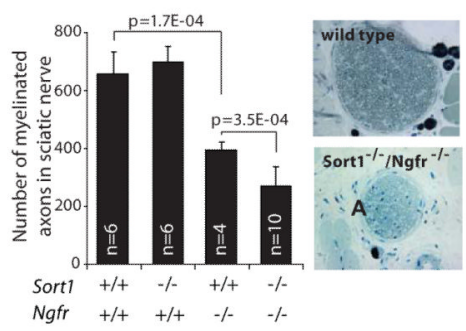

b

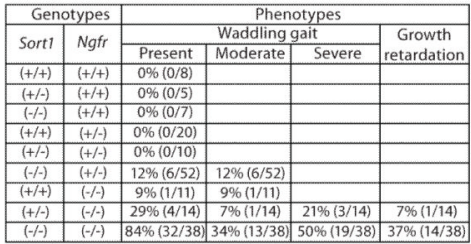

d

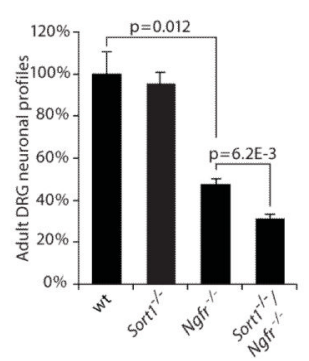

Figure 4.

Abnormal gait and peripheral neuropathy in sortilin and $\mathrm{p} 75^{\mathrm{NTR}}$ double knockout mice a, Abnormal hind-limb posture in $\mathrm{Sort}^{-1-} / \mathrm{Ngfr}^{-/-}$mice. b. Correlation between genotype and penetrance of the various phenotypes. Parenthesis indicates affected animals of the total number of mice in each group. c, Myelinated axon count in the sciatic nerve (left) and corresponding nerve morphology (right). d, Number of neurons in adult L4 and L5 DRG $(n=4)$. 
a

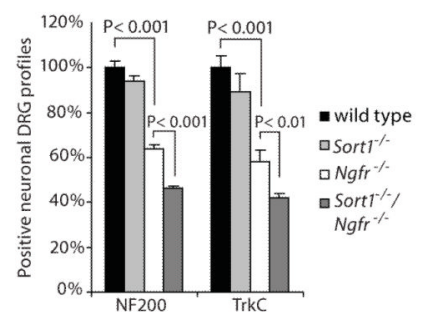

C

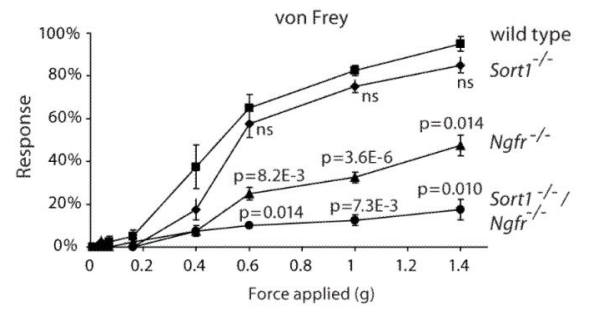

b

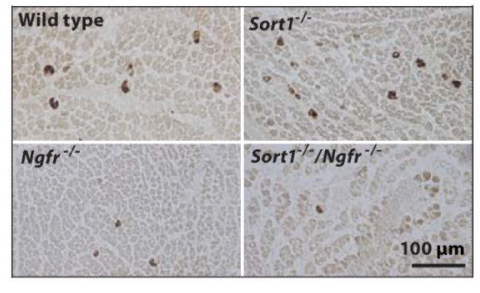

d

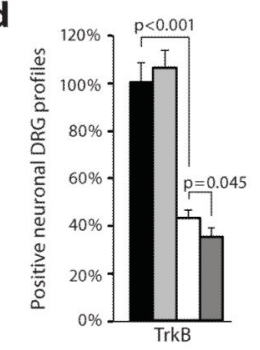

Figure 5.

Loss of sortilin aggravates TrkC and TrkB phenotypes in $\mathrm{p} 75^{\mathrm{NTR}}$ knockout mice. a, Quantification of proprioceptive neurons in L4/L5 DRGs using markers for NF200 and $\operatorname{TrkC}(\mathrm{n}=8)$. b, Reduction of muscle spindles in hind-limbs in P1 Sort $1^{-/-} / \mathrm{Ngfr}^{-/-}$mice. c, von Frey stimulus-response curves $(\mathrm{n}=4)$. The $\mathrm{p}$-values are relative to wt (Sort ${ }^{-1-}$ and $\mathrm{Ngfr}^{-1-}$ curves) or $\mathrm{Ngfr}^{-/-}\left(\mathrm{Sort}^{-1-} / \mathrm{Ngfr}^{-/-}\right.$curve $)$. d, Numbers of TrkB positive DRG neurons in adult mice $(\mathrm{n}=8)$. 
a

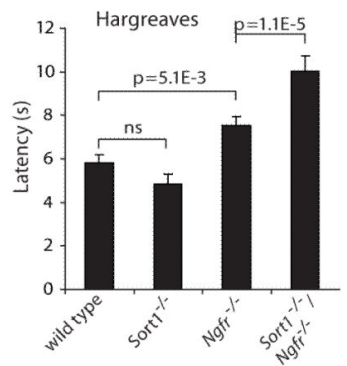

C

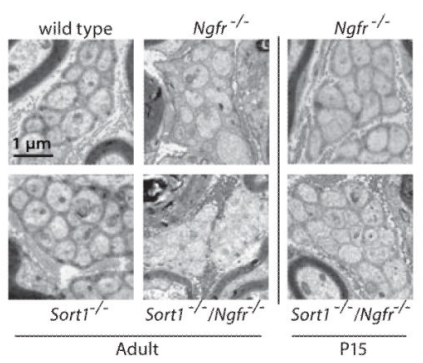

b

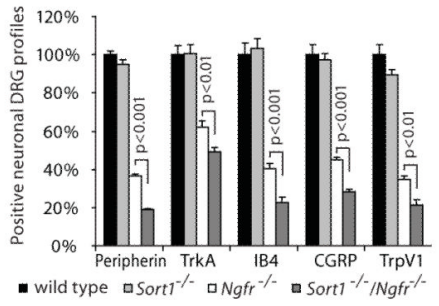

d

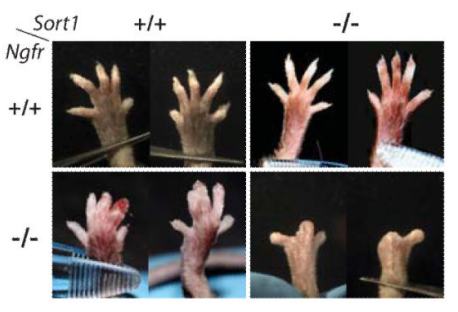

Figure 6.

Sortilin deficiency aggravates TrkA phenotypes in $\mathrm{p} 75^{\mathrm{NTR}}$ knockout mice. a, Thermal response as measured by Hargreaves test $(n=10)$. b, Quantification of nociceptive neuron subtypes in L4-L5 DRG ( $\mathrm{n}=8$ ). c, Electron micrographs showing the morphology of Remak bundle fibers in adult and P15 mice. d, Hind limb degeneration in DKO at 3-4 month of age. 
a
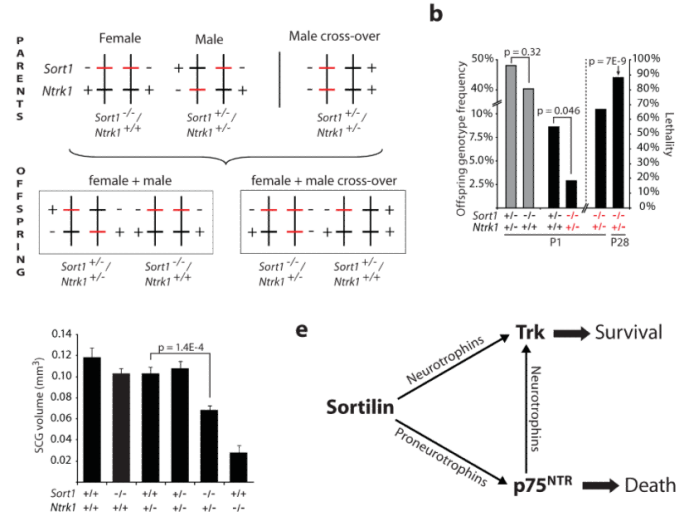

e
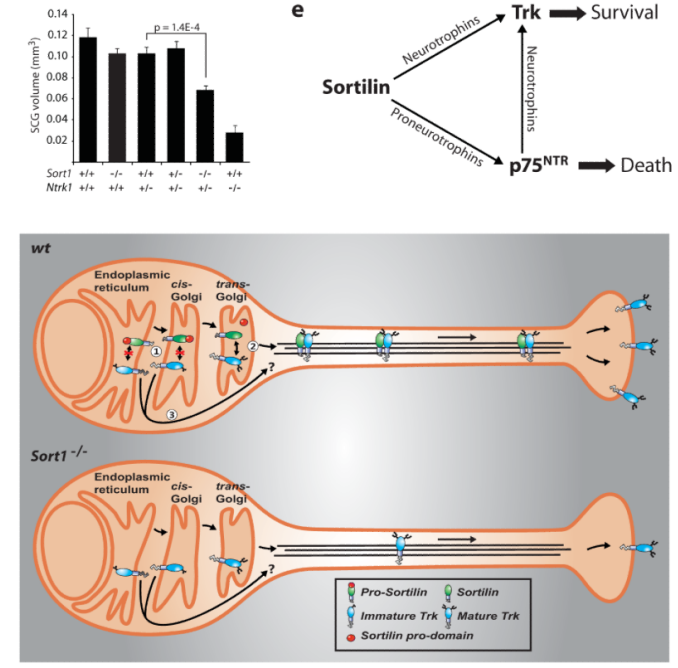

Figure 7.

Absent sortilin expression induces TrkA phenotypes in $N$ trk $1^{+/-}$mice. a, Schematic representation of possible genotypes in offspring from Sort $1^{+/-} / \mathrm{Ntrk}^{+/-}$and Sort $1^{-/-}$/ Ntrk $1^{+/+}$breeding. b. Genotype frequency of offspring at P1 (left side of dashed line) and

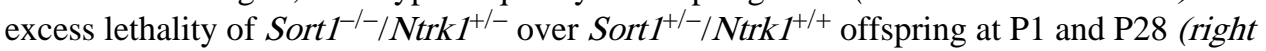
side of dashed line), cf. panel a. c, SCG volume in newborns of the indicated genotypes. d, A schematic model describing sortilin in Trk trafficking. 1) Trk receptors are unable to bind pro-sortilin in the ER and cis-Golgi compartment; 2) following furin-mediated activation of sortilin in the TGN sortilin binds Trk and facilitates its anterograde transport; 3 ) immature and incompletely glycosylated Trk receptors segregate from the biosynthetic pathway prior to activation of sortilin. Lower panel: Reduced axonal targeting of $140 \mathrm{kDa}$ mature Trk but preserved transport of the immature $110 \mathrm{kDa}$ form in sortilin knockout neurons. $\mathbf{e}$, Schematic model of the 'Neurotrophin triangle' concept. 